\title{
Gestión eficiente del agua con los contadores ultrasónicos inteligentes YZATEC
}
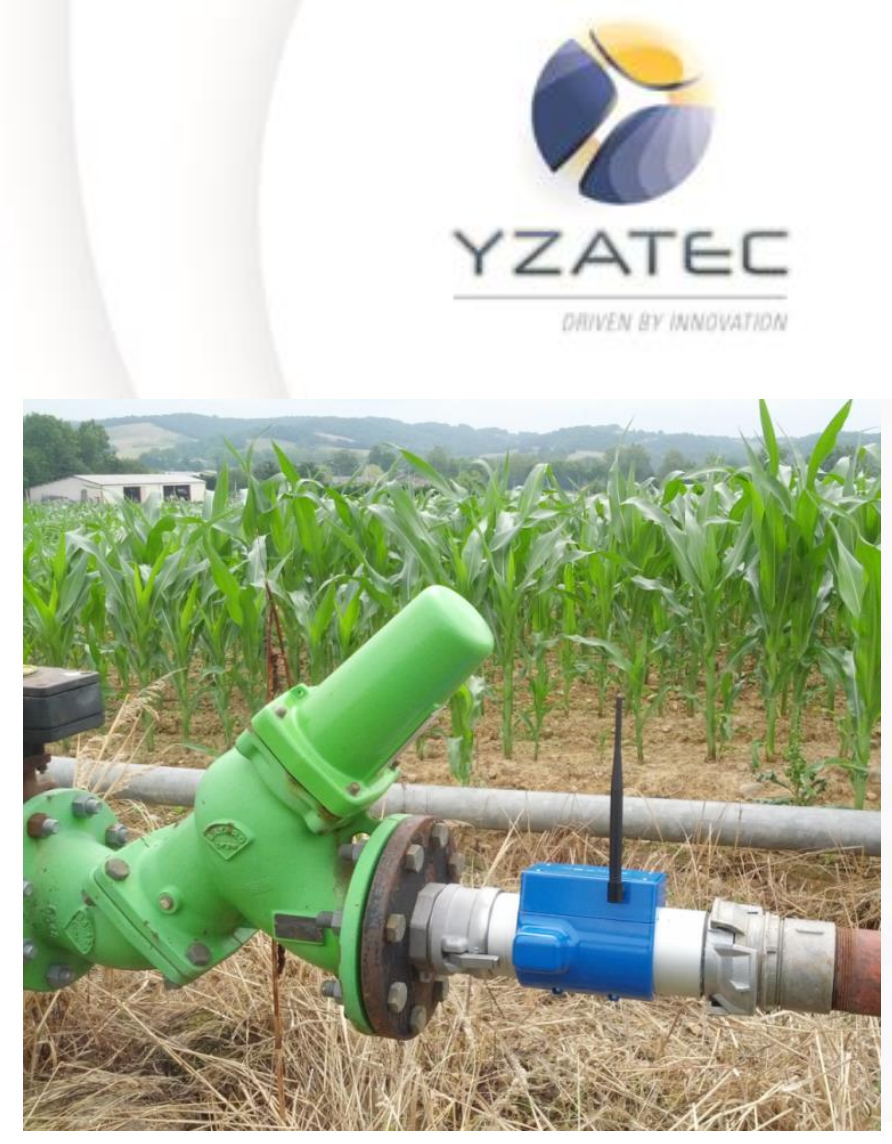
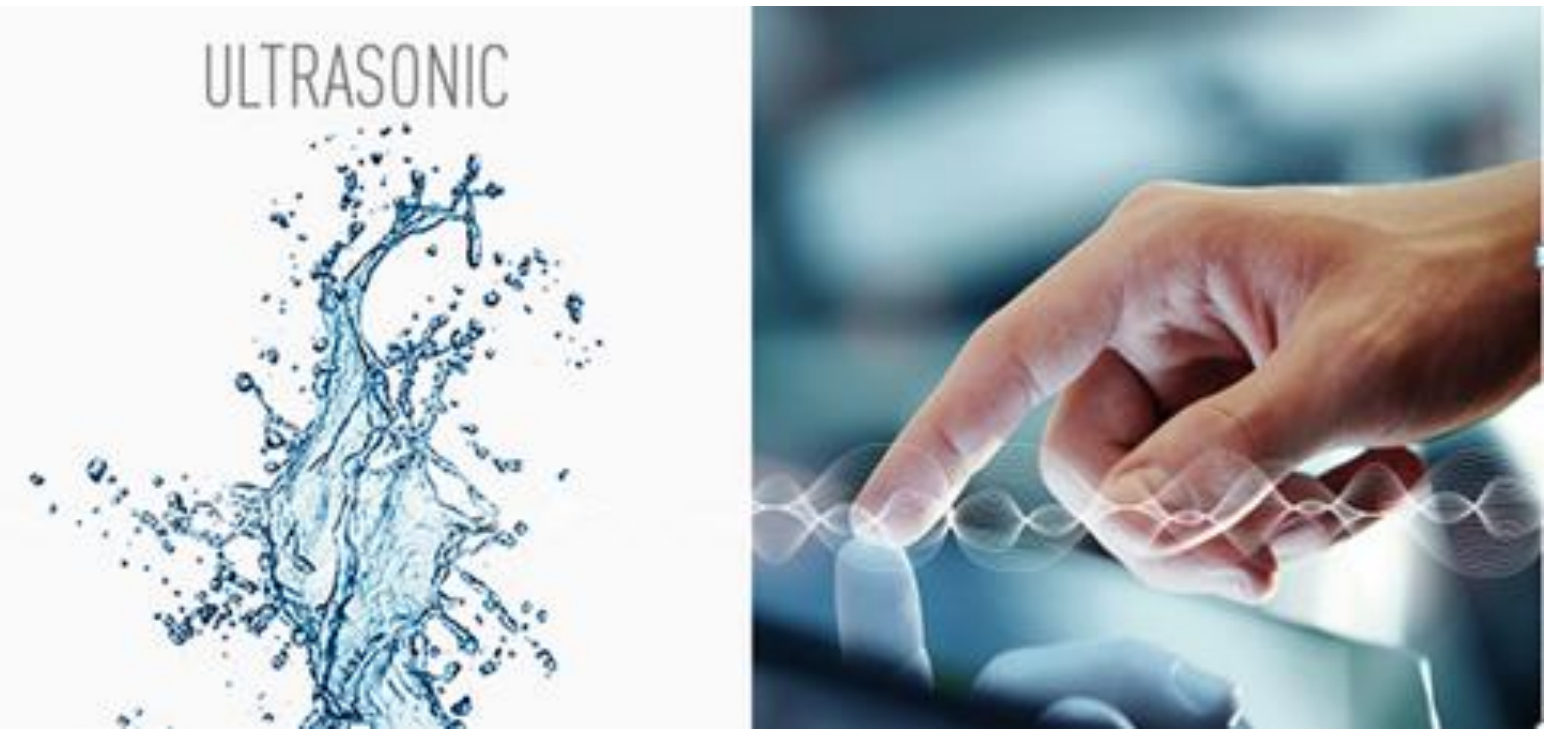

DOI: http://dx.doi.org/10.21151/CNRiegos.2016.TC3 
1/ Problemas en redes de distribución de agua

2/ Nueva gestión con los contadores ultrasónicos inteligentes

3/ Yzatec : simple desde la instalación hasta la gestión Como funciona

Como se instala

Como se gestiona

4/ Mejorar el rendimiento de su cultivo

Reducir los costes de mantenimiento

Gestión inteligente de su regadío

Facturar y pagar su consumo real de agua

5/ CACG, ejemplo de optimización de riego 
1- Problemas en redes de distribución de agua YZATEC ORIVEN SY INNOVATION 


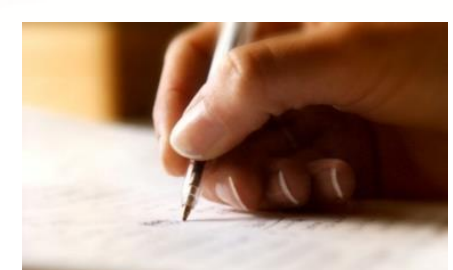

> Lectura manual o sistema poco compacto, varios interlocutores, costoso

$>$ Abono químicos corosivos $=>$ deterioro de los contadores

$>$ Elevada pérdida de carga => aumenta el coste energético

$>$ Pérdida de precisión por uso => información errónea del consumo

$>$ No miden caudales pequeños $=>$ consumo de agua no facturado

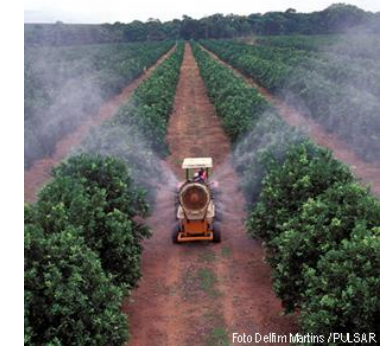

$>$ Obstrucción del contador $=>$ coste de mantenimiento

$>$ Funcionamiento erroneo en posición vertical
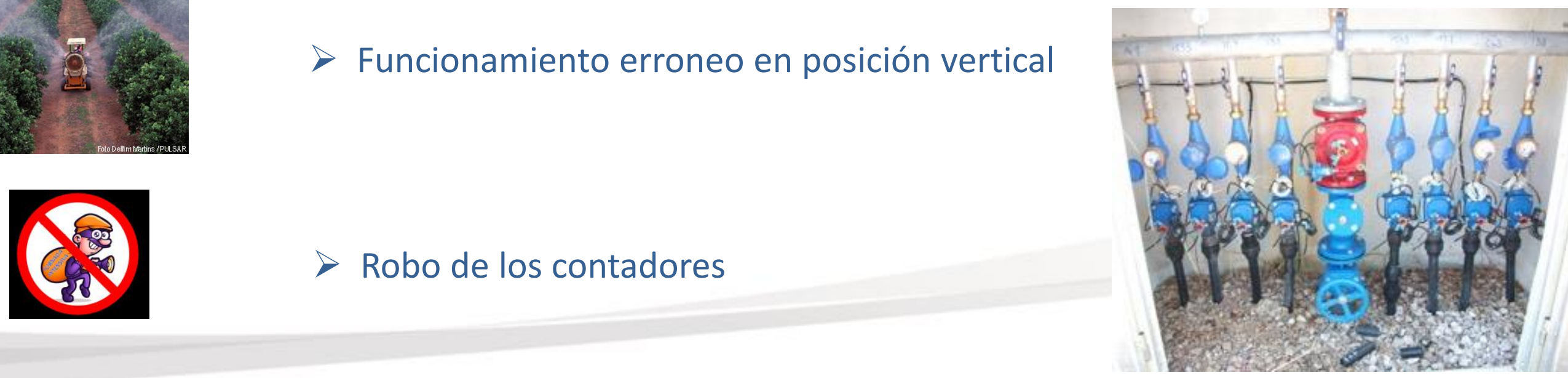
YZATEC 2- Nueva gestión con los contadores ultrasónicos 
Sin pérdida de carga, permiten obtener un significativo ahorro energético

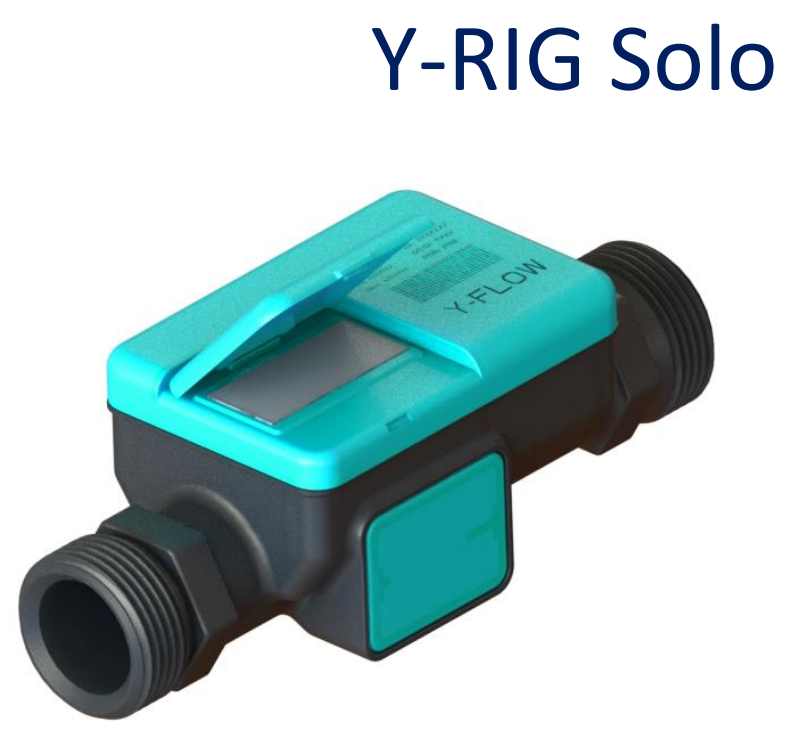

Medición no manipulable (con imanes u otros objetos)

Medición del consumo real del agua

Muy resistente y compacto

Instalación vertical y horizontal

Ahorro en coste de mantenimiento 
Sin pérdida de carga, permiten obtener un significativo ahorro energético

$$
\text { Y-RIG }
$$

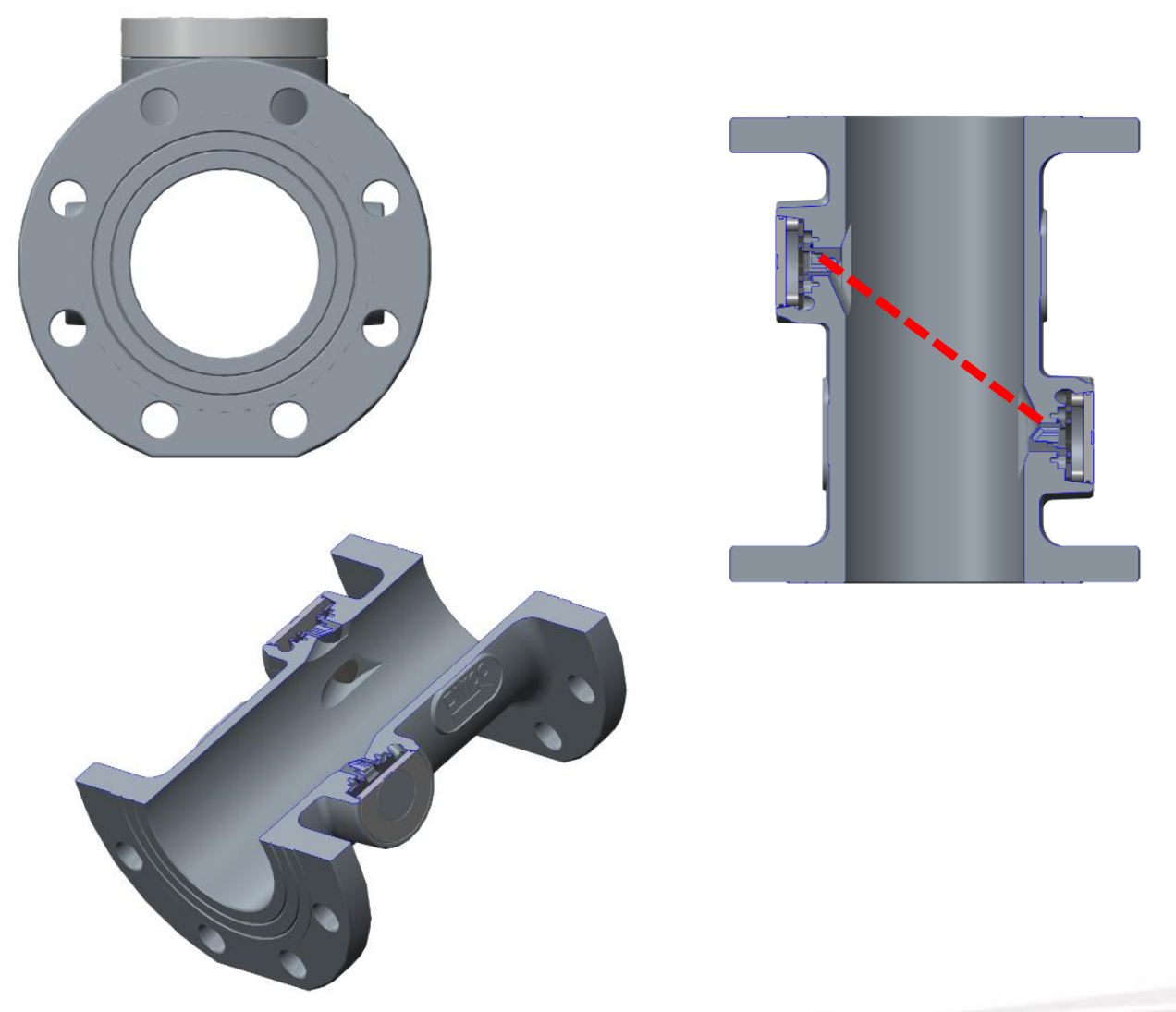

Medición no manipulable (con imanes u otros objetos)

Medición del consumo real del agua

Muy resistente y compacto

Gestión de su consumo a distancia

Ahorro en coste de mantenimiento 
3- Yzatec : simple desde la instalación hasta la gestión YZATEC 


\section{Plug \& Play}
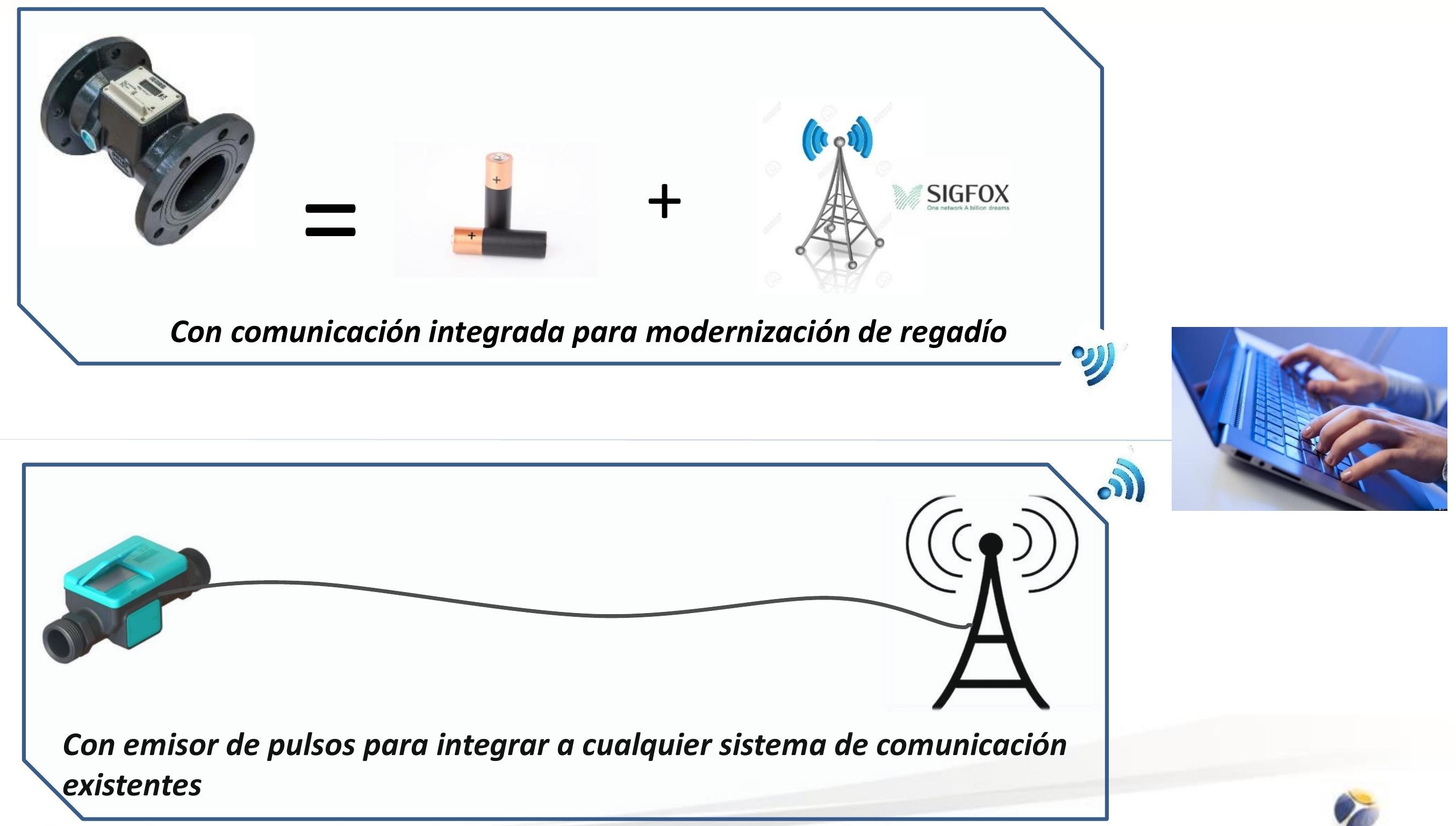


\section{Arquitectura Y-RIG con red SIGFOX}

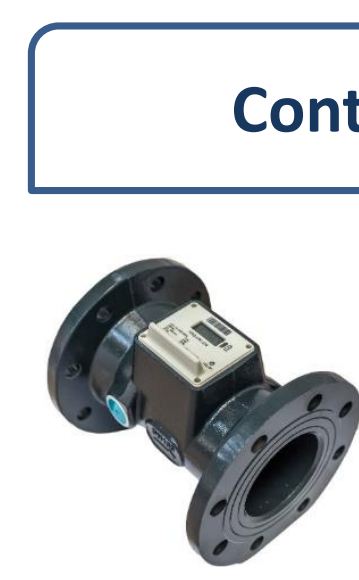

Y-RIG DN 100 Riego

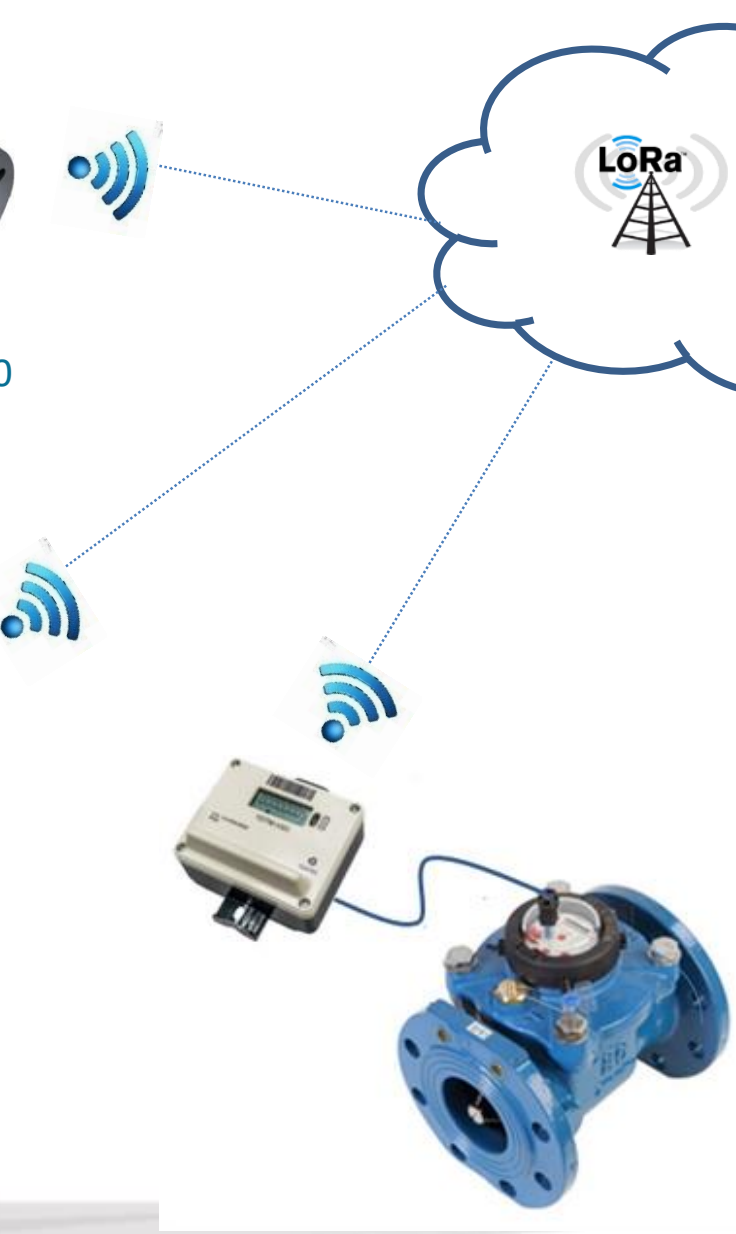

Red



Y-RIG DN 50 Riego

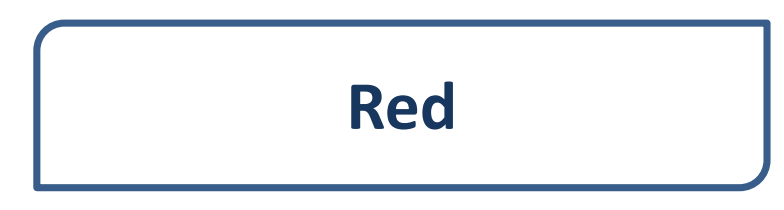

\section{Plataforma web de telegestión}

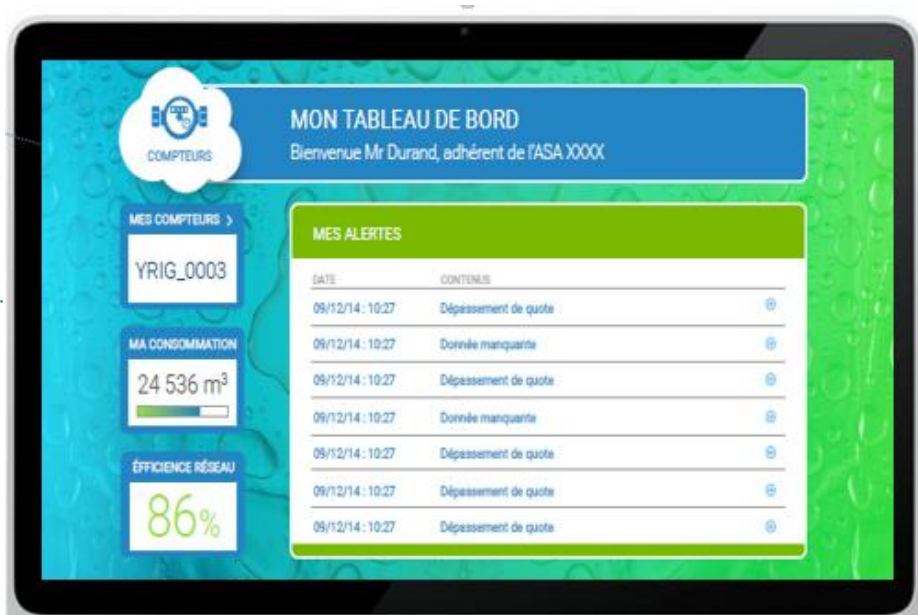

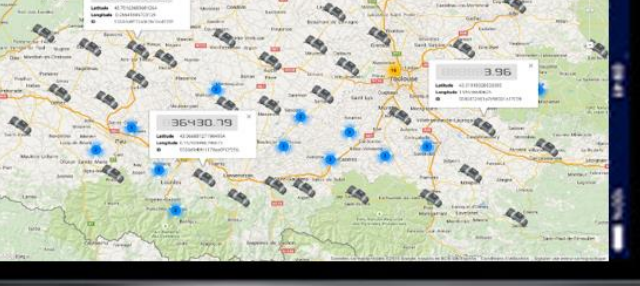

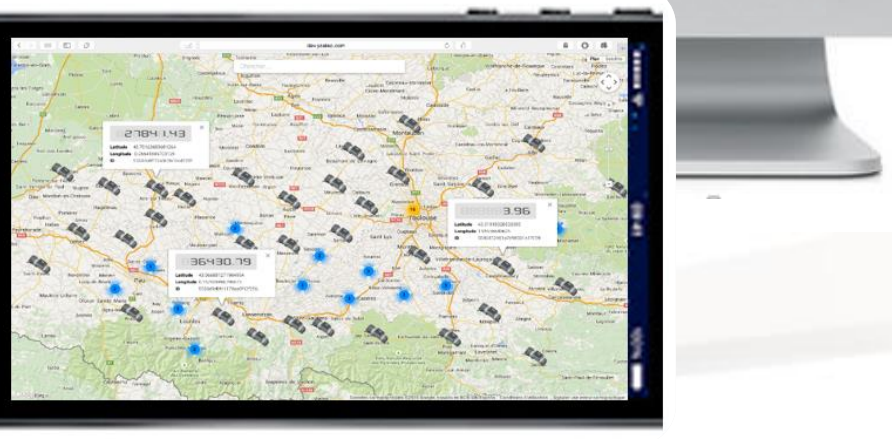




\section{Aceso simple a sus datos de consumo}
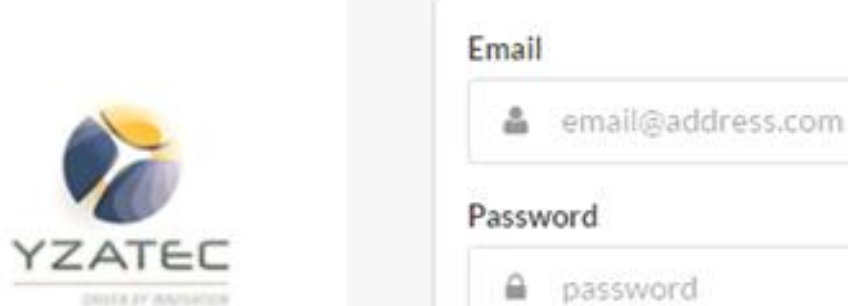


\section{Almacenamiento del historico de consumo}

8 demonstrator - 15-ccol-c $x$

$\leftarrow$ C
$*$ Apps

$\equiv$

Last messages 15-CC01-0063

\begin{tabular}{|c|c|c|c|c|c|}
\hline \# & 苗 & 11III & 28 & ○ & 뜨 \\
\hline 2738 & 5/2/2016, 8:56:03 AM & $15-\mathrm{CC} 01-0063$ & 474.74 & $30: 41: 05$ & 21.89 \\
\hline 2737 & 5/2/2016, 7:55:05 AM & $15-\mathrm{CC} 01-0063$ & 458.88 & 29:57:37 & 0.00 \\
\hline 2736 & 5/2/2016, 6:56:03 AM & $15-\mathrm{CC} 01-0063$ & 458.88 & 29:57:37 & 0.00 \\
\hline 2735 & 5/2/2016, 5:55:03 AM & $15-\mathrm{CC} 01-0063$ & 458.88 & $29: 57: 37$ & 0.00 \\
\hline 2734 & 5/2/2016, 4:56:03 AM & $15-\mathrm{CCO1}-0063$ & 458.88 & $29: 57: 37$ & 0.00 \\
\hline 2733 & 5/2/2016, 3:55:03 AM & $15-\mathrm{CC} 01-0063$ & 458.88 & $29: 57: 37$ & 0.00 \\
\hline 2732 & 5/2/2016, 2:56:03^M & $15-\mathrm{CC} 01-0063$ & 158.88 & 29:57:37 & 0.00 \\
\hline 2731 & 5/2/2016, 1:55:03 AM & $15-\mathrm{CC} 01-0063$ & 458.88 & $29: 57: 37$ & 0.00 \\
\hline 2730 & 5/2/2016. 12:56:03 AM & $15-\mathrm{CC} 01-0063$ & 458.88 & 29:57:37 & 0.00 \\
\hline 2729 & 5/1/2016, 11:55:03 PM & $15-\mathrm{CCO}-0063$ & 450.00 & $29: 57: 37$ & 0.00 \\
\hline 2728 & 5/1/2016, 10:56:03 PM & $15-\mathrm{CC} 01-0063$ & 458.88 & $29: 57: 37$ & 0.00 \\
\hline 2727 & 5/1/2016, 9:55:03 PM & $15-\operatorname{Cr.01-00633~}$ & 458.88 & 29:57:37 & 0.00 \\
\hline 2726 & 5/1/2016,8:56:04 PM & $15-C C 01-0063$ & 458.88 & $29: 57: 37$ & 0.00 \\
\hline 2725 & 5/1/2016, 7:55:04 PM & $15-\mathrm{CC} 01-0063$ & 458.88 & 29:57:37 & 0.00 \\
\hline 2721 & 5/1/2016,6:56:01 PM & $15-\mathrm{CC} 01-0063$ & 158.88 & 29:57:37 & 0.00 \\
\hline 2723 & 5/1/2016, 5:55:04 PM & 15-CC01-0063 & 458.88 & 29:57:37 & 0.00 \\
\hline 2722 & 5/1/2016.4:56:04 PM & $15-\mathrm{CC} 01-0063$ & 458.88 & $29: 57: 37$ & 0.00 \\
\hline 2721 & 5/1/2016, 3:55:03 PM & $15-\mathrm{cC} 01-0063$ & 450.00 & $29: 57: 37$ & 0.00 \\
\hline
\end{tabular}




\section{Y-RIG}

\section{Gestión Diaría de su Regadío}

- Seguir su consumo a distancia

- Lectura global con el display

- Posibilidad de analizar los datos

\section{Poco Mantenimiento}

- Parte comunicante integrada

- Sin obstrucción

\section{Ahorro Agua e Energía}

- Sin pérdida de carga

- Detecta fugas

- Funciona con dos pilas (10 años)

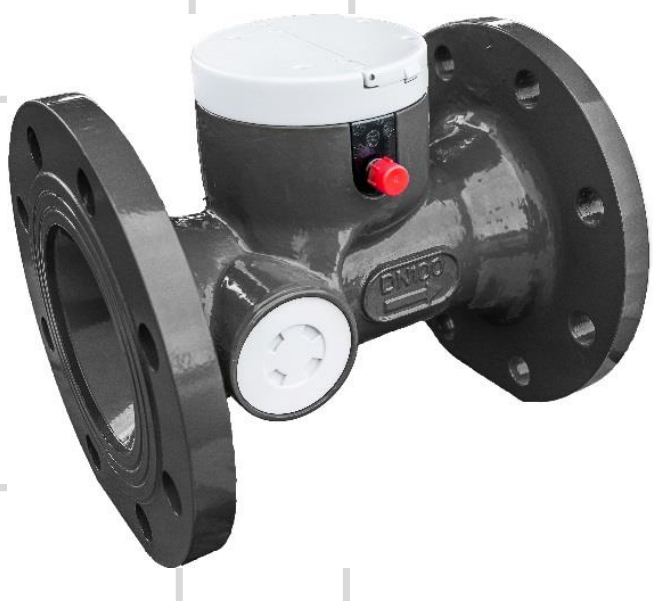

\section{Instalación}

- Se adapta a las instalaciones existentes

- Se pueden poner tubos simples

- Instalación UO DO 
4- Mejorar el rendimiento de su cultivo: Reducir los costes de mantenimiento Gestión inteligente de su regadío Facturar y pagar el consumo real de agua 
$\checkmark$ Instalación de la Comunicación + Fuente de Energía + Contador = en una Vez

$\checkmark$ Sin Parte Mecánica

$\checkmark$ Medición Precisa en Bajos y Altos Caudales

$\checkmark$ Resistente a las Condiciones de Uso Agrícola
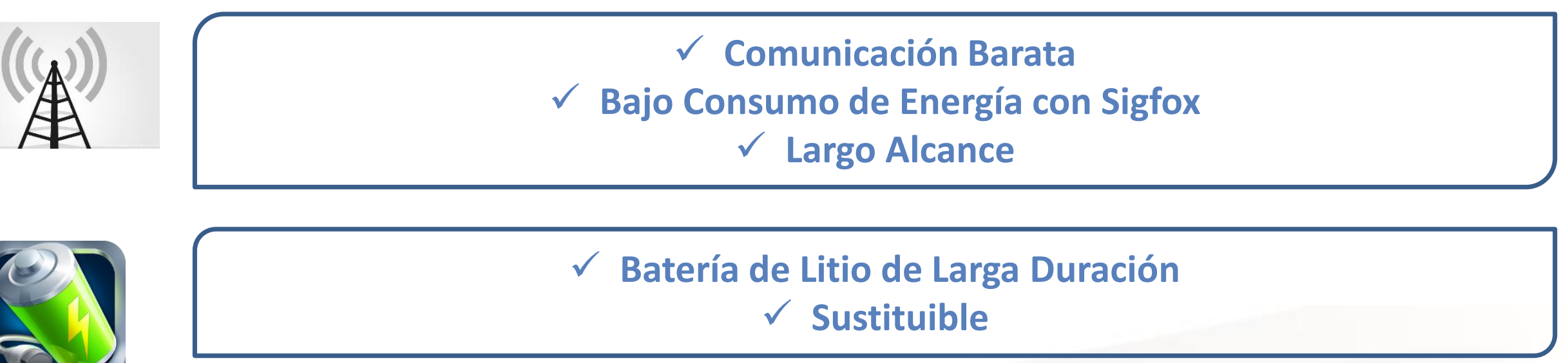
$\checkmark$ Bajo Consumo de Energía con Sigfox
$\checkmark$ Largo Alcance

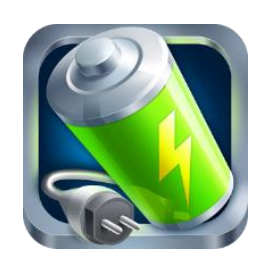

\author{
$\checkmark$ Sustituible
}




\section{Una gama completa en plástico y en fundición}

\section{DATOS DE RENDIMIENTO}

DN 50

DN 65

DN 80

DN 100

DN 125

DN 150

\begin{tabular}{|c|c|c|c|c|c|}
\hline DN & 50 & 65 & 80 & 100 & 125 \\
\hline Q4 Caudal máximo (m3/h) & 80 & 125 & 125 & 200 & 320 \\
\hline Q3 Caudal nominal (m3/h) & 63 & 100 & 100 & 160 & 250 \\
\hline Q2 Caudal de transición (m3/h) & 0,30 & 0,50 & 0,50 & 0,80 & 1,30 \\
\hline Q1 Caudal mínimo (m3/h) & 0,20 & 0,32 & 0,32 & 0,50 & 0,80 \\
\hline Ratio (Q3/01) & 250 & 250 & 250 & 250 & 250 \\
\hline Pérdida de presión en la Q3 (bar) & & & $<0.05$ & & \\
\hline
\end{tabular}

DN 20

DN 25

DN 32

DN 40 
5- CACG, ejemplo de optimización de riego 


\section{CACG}

\section{CONTEXTO}

CACG es la mayor comunidad de riegos en Francia

15.000 puntos de medición

Despliegue de Y-RIG 2015/2016

Diseñado para mejorar el control del consumo

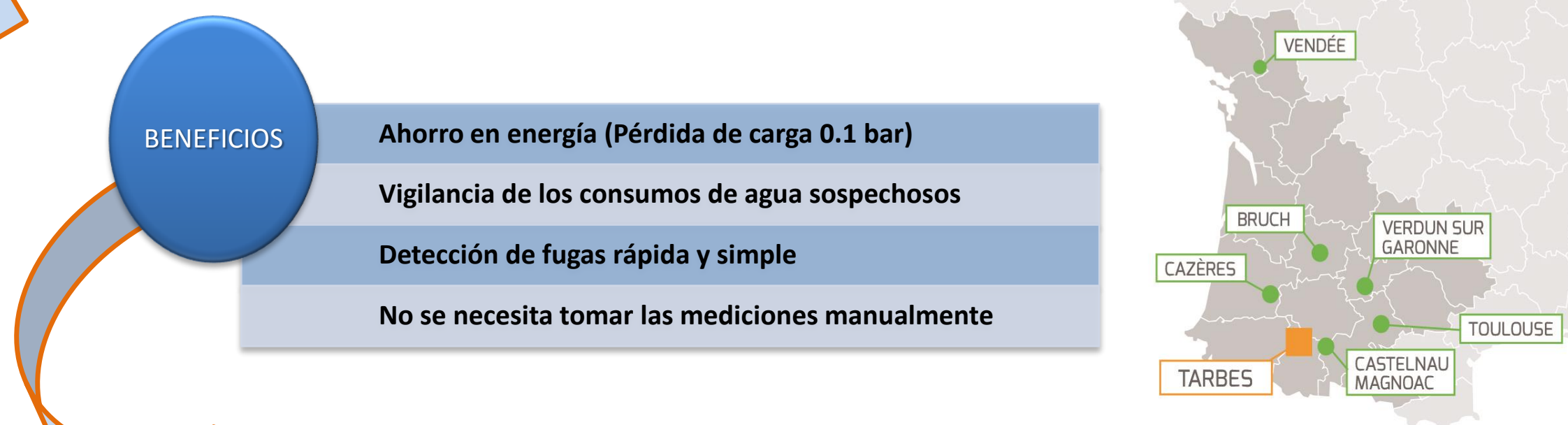

\section{Retorno de la inversión < 1 año}




\section{Sede y delegaciones en España}

\section{Levante :}

victor.goncalves@yzatec.com

Tel. 722528900

Andalucia/Norte España : alberto.calle@yzatec.com

Tel.617577815

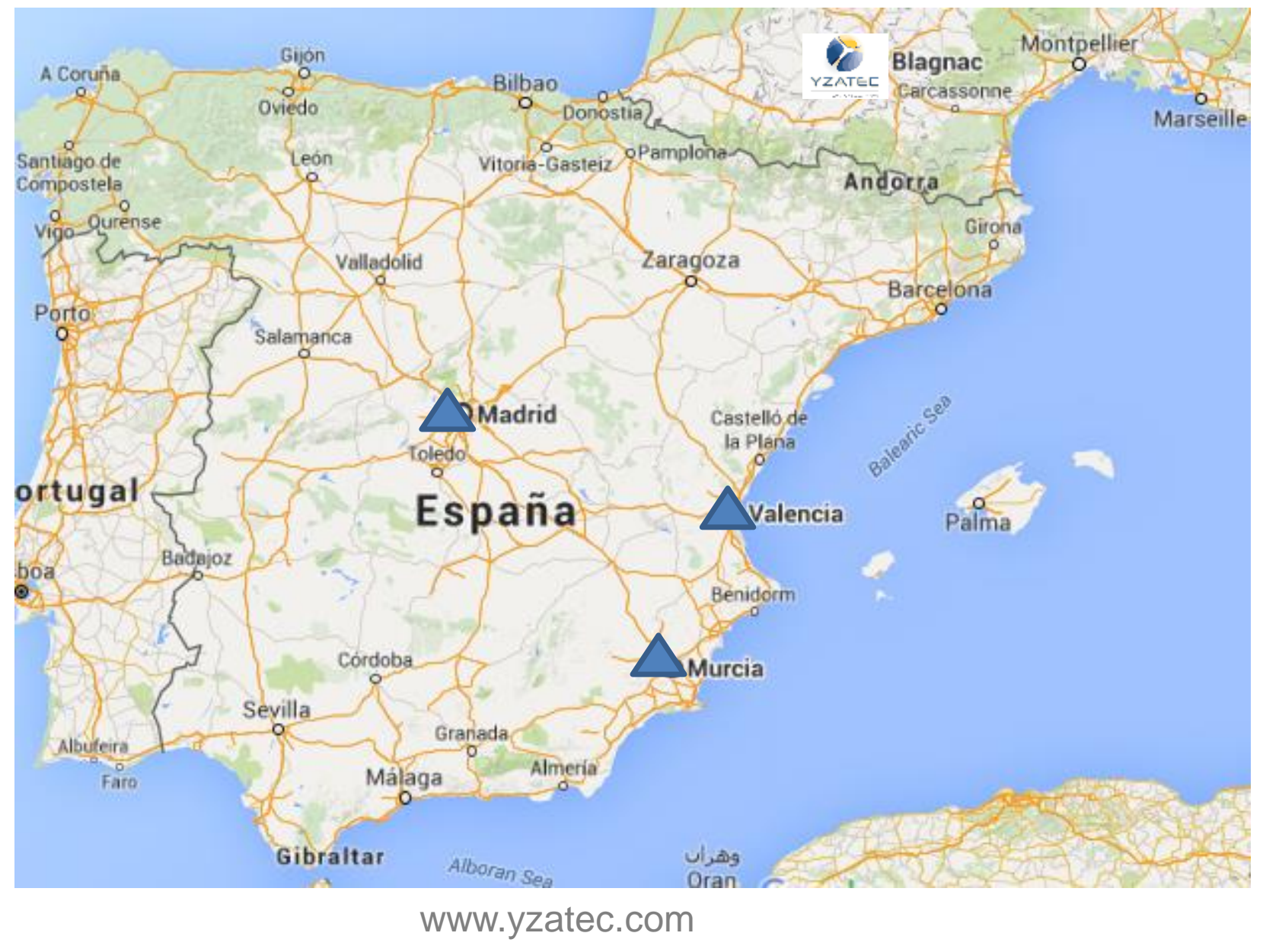

